\title{
Texture Design for Reducing Tactile Friction Independent of Sliding Orientation on Stainless Steel Sheet
}

\author{
S. Zhang ${ }^{1,2}$ (1) X. Zeng ${ }^{1,3} \cdot$ A. Igartua $^{4} \cdot$ E. Rodriguez-Vidal ${ }^{4}$ E. van der Heide Re $^{1,5}$
}

Received: 16 February 2017/Accepted: 11 May 2017/Published online: 27 May 2017

(C) The Author(s) 2017. This article is an open access publication

\begin{abstract}
Surface texture is important for contact mechanical and tribological phenomena such as the contact area and friction. In this research, three different types of geometrical microstructures were designed and fabricated by pulsed laser surface texturing as semi-symmetric (grooved channel), asymmetric fractal (Hilbert curve), and symmetric patterns (grid). A conventionally finished surface as a reference sample from the same stainless steel sheet material was compared. From the experimental approach, a multiaxis force/torque transducer was used to investigate the functionality of surface texture based on measuring the tactile friction in three different sliding directions: perpendicular, parallel, and $45^{\circ}$ to the textures. According to the dynamic friction measurements, the grid texture was indeed orientation independent. The other samples showed orientation-dependent frictional behavior, especially the grooved channel texture and reference sample. Furthermore, an analytical approach was applied to estimate the values of the friction coefficient by the pressure distribution method. From both the experimental and analytical approaches, the grid pattern was validated to be
\end{abstract}

S. Zhang

s.zhang@utwente.nl

1 Laboratory for Surface Technology and Tribology, Faculty of Engineering Technology, University of Twente, Drienerlolaan 5, 7522 NB Enschede, The Netherlands

2 State Key Laboratory of Tribology, Tsinghua University, Beijing 100084, China

3 Shanghai Advanced Research Institute, Chinese Academy of Sciences, Beijing, China

4 IK4-Tekniker, C/Ignacio Goenaga, 20600 Eibar, Spain

5 Faculty of Civil Engineering and Geosciences, TU Delft, Stevinweg 1, 2628 CN Delft, The Netherlands the optimal texture design in the concern of friction reduction and orientation-independent behavior.

Keywords Sliding - Surface texture - Microfabrication · Skin friction

\section{Introduction}

Tactile friction, or skin friction, is a resistive force that prevents the skin from sliding freely against the countersurface. Whether putting the clothes on in the morning, texting the message on the cell phone, or playing a musical instrument to entertain yourself, skin friction is inevitable and plays an important role in tactile perception. In the modern market, touch sensation was not forgotten or underestimated. In fact, great concerns in skin friction, especially fingertip skin friction, were raised in relation to the feel of products to the consumers [7]. The dynamic friction between the fingertip and counter-body determines how we perceive a surface, which can sway the consumers' judgments toward the lucrative products [4].

In skin tribology, many experimental studies of the friction contacts between skin and different objects were conducted with in vivo measurements [20, 23, 30, 33, 37]. As a multilayered biomaterial, skin consists of stratum corneum (SC), epidermis, dermis, and hypodermis (subcutaneous tissues) [32, 38]. Each skin layer has different mechanical properties associated with different composition, thickness, and hydration degree. From the literature, the frictional behavior of skin is influenced by the complex interaction of the material and surface properties of the counter-body and the skin itself [18, 21]. From the mechanical perspective, the microscaled geometric structure of texture can affect the friction between the skin and 
counter-surface. Groove patterns, for example, as the most common geometric structure, were studied and found to have the ability of reducing the tactile friction during fingertip exploration [31]. Recent studies are focused on the influential factors like the material properties, skin condition, and contact parameters of the counter-body and the skin [27, 35].

The real contact area plays a pivotal role in skin friction. According to the research, skin friction decreases with the reduction in the real contact area [24, 39]. In skin tribology, both the adhesion and deformation components of friction (two-term model of friction) contribute to tactile friction $[8,9]$. However, the adhesion component of friction (refer to Eq. 1) plays the dominant role $[1,11]$.

$F_{\text {f,adh }}=\tau A_{\text {real }}$

where $F_{\mathrm{f}, \text { adh }}$ represents the adhesion component of friction; $A_{\text {real }}$ is the real contact area; $\tau$ is the interfacial shear strength. The real contact area is significantly less than the apparent contact area when skin is interacting with the microstructured counter-surface. For the interfacial shear strength $\tau$, it has been found to have a linear function of the average contact pressure $(p)$ as:

$\tau=\tau_{0}+\alpha \bar{p}$

where $\tau_{0}$ is the intrinsic interfacial shear strength; $\alpha$ is the pressure coefficient. After combining Eqs. (1) and (2), the coefficient of friction can be expressed as follows:

$\mu=\frac{F_{\mathrm{f}, \text { adh }}}{F_{\mathrm{N}}}=\frac{\tau}{\bar{p}}=\frac{\tau_{0} A_{\text {real }}}{F_{\mathrm{N}}}+\alpha$

The adhesion component of friction is directly related to the real contact area, and the reduction in real contact area can greatly decrease friction.

Stainless steel sheet has a wide variety of applications in industry and science, especially for automotive and domestic appliances. The investigation of tactile friction on the textured surface can enhance the understanding of tactile perception, and the human-product interactions of stainless steel products will be improved [41]. Surface textures are widely applied in studies on dynamic friction to reduce the adhesive component of friction, and most of them were carried out under unidirectional sliding conditions [5, 17]. However, from our previous work [39], the orientation effect has great influence on the skin friction between fingertip and textured counter-surface [39]. An experimental approach was used based on measuring tactile friction on textured stainless steel sample with grooved channel. A polished surface from the same stainless steel was compared as a reference sample. In vivo measurements revealed that sliding orientation greatly influenced the frictional performance on grooved channel texture compared to the reference sample. Moreover, parallel sliding showed a higher dependency of friction coefficient than perpendicular sliding on the depth of the grooved samples.

In this paper, the deterministic and stochastic surface textures are used for friction experiments. The stochastic sample is one conventionally finished $2 \mathrm{G}$ surface as the reference (sample Z000). The three deterministic surface textures are fabricated on the sample reference material based on their geometric types. Each one is commonly used in its category: grooved channel as a semi-symmetric pattern (sample Z001), Hilbert curve as an asymmetric fractal pattern (sample Z002), and pillar as a symmetric pattern (sample Z003). Based on the experimental approach, the tactile frictions on each sample in different sliding directions were measured. The objective is to design a surface texture which not only decreases the friction but also eliminates the orientation effect.

\section{Textural Design and Materials}

\subsection{Friction Model and Measurement}

For the friction measurements, each specimen was fixated on the top of a load cell (ATI Gamma six-axis force/torque transducer, ATI Industrial Automation, Apex, NC, USA; refer to Fig. 1). The resolution of the force measurement is $25 \mathrm{mN}$ in normal direction (z-axis) and $12.5 \mathrm{mN}$ in tangential direction ( $x$ - and $y$-axes). Each experiment consisted of five sliding repetitions toward the body. Three sliding directions were performed: $0^{\circ}$ (parallel), $45^{\circ}$, and $90^{\circ}$ (perpendicular) to the surface texture. The contact finger (right hand index finger) used in this experiment is from a 32-year-old male. The fingertip was washed by soap and air-dried, and the stainless steel samples were cleaned by ethanol before each measurement. The environmental conditions of the laboratory were $20 \pm 1{ }^{\circ} \mathrm{C}$ as room temperature and $40 \pm 5 \%$ as relative humidity. The hydration level of the testing finger was measured by Corneometer CM 825 (Courage + Khazaka GmbH, Germany) at $32 \pm 3$ AU. During the dynamic skin friction measurements, the testing finger was in contact with the specimen, and sliding occurred at a steady velocity with an average of $17 \pm 4 \mathrm{~mm} / \mathrm{s}$. The sampling rate was $100 \mathrm{~Hz}$. Followed by the same procedure, the applied normal load, friction force, and coefficient of friction (COF) were recorded by the ATI transducer.

\subsection{Textural Design of Geometry}

The grooved channel pattern is expected to be influenced by the orientation effect. The distribution of geometric structures is different in the perpendicular sliding direction compared to the parallel and $45^{\circ}$ sliding directions, as 
Fig. 1 a Three-axis

force/torque transducer (ATI Industrial Automation, Apex, NC, USA); b skin friction measurement

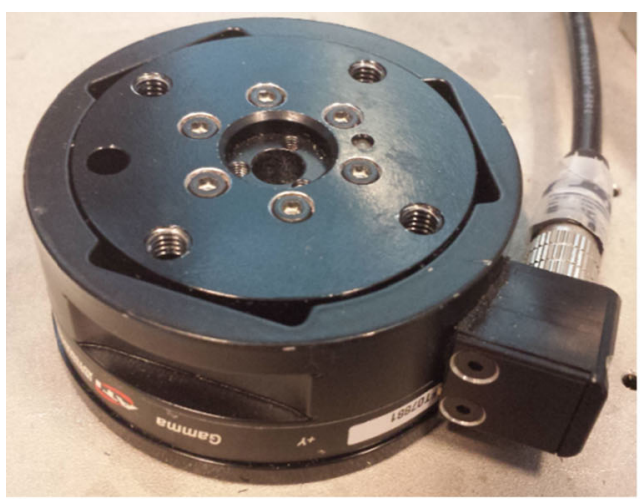

(a)

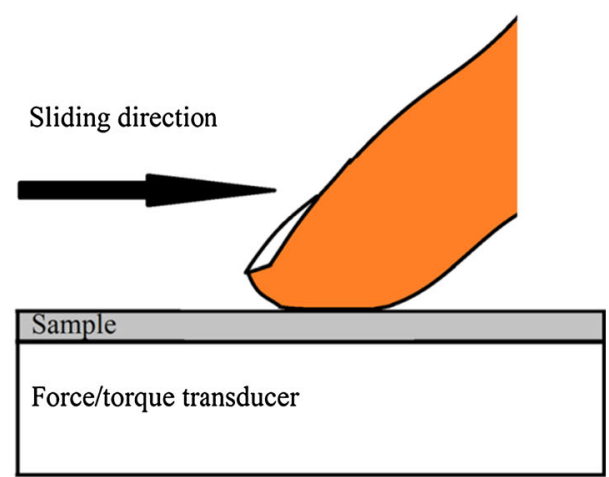

(b)
Fig. 2 Parallel, $45^{\circ}$, and perpendicular sliding direction on a grooved channel, b Hilbert curve, and $\mathbf{c}$ pillar texture
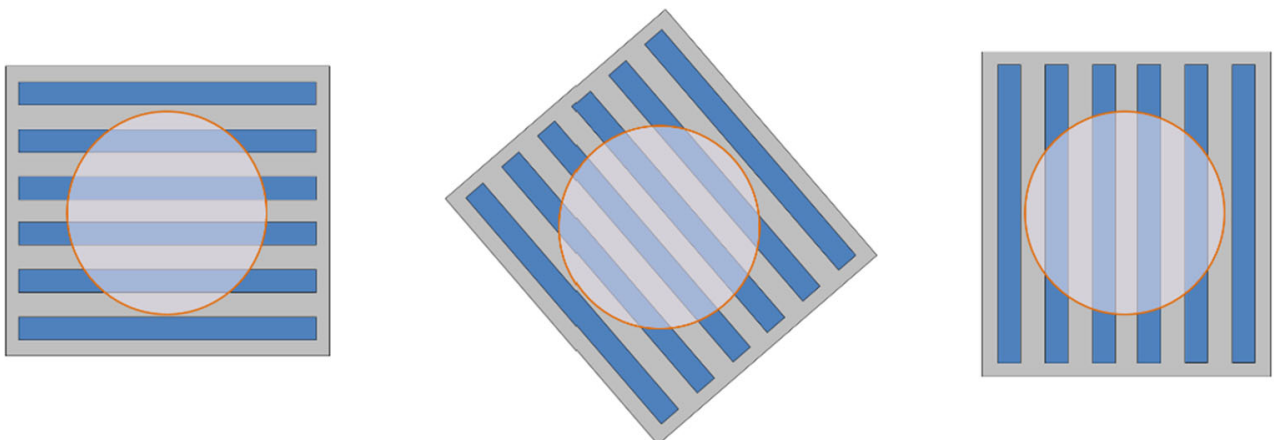

(a)
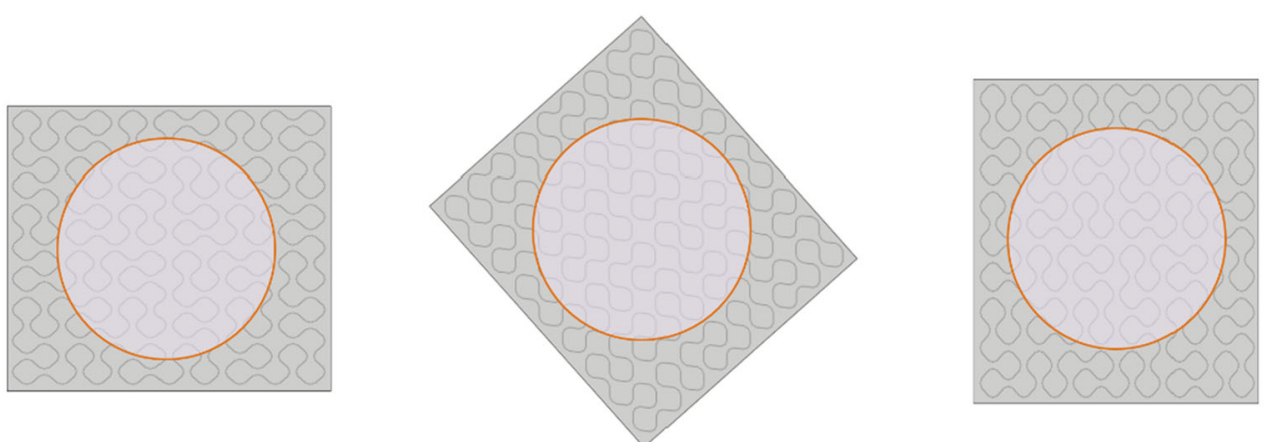

(b)

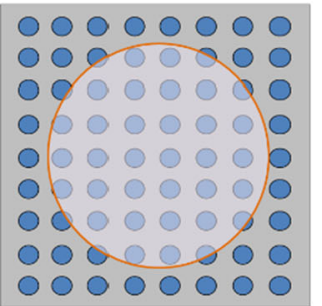

000000000 000000000 000000000 000000000 000000000 000000000 000000000 000000000 
shown in Fig. 2a. The area in contact changes accordingly. The change of pattern layout in different sliding direction is able to alter the contact area and friction between the skin and grooved channel texture. Unlike the grooved channel pattern, the Hilbert curve (asymmetric fractal pattern) demonstrates the ability to contain the entire two-dimensional unit space by a peculiar geometric structure which expands in all directions [2, 3, 13, 15, 25]. As shown in Fig. 2b, the areas in contact with different sliding directions have the similar pattern layout. In this research, the stroke length is approximately the length of the sample size which is $40 \mathrm{~mm}$. With this stroke length, the overall encountered peaks and valleys are self-similar across different direction which gives the ability to minimize the effect of orientation. On the other hand, the pillar texture is a symmetric geometric structure which has an identical pattern distribution in $x$-axis and $y$-axis directions. As shown in Fig. 2c, the total number of pillars in contact was nearly same for all three sliding directions. The parallel sliding direction has approximately 33 counts of pillars in contact; the $45^{\circ}$ sliding direction has approximately 34 pillars in contact; the horizontal sliding direction has approximately 34 pillars in contact with the same unit of contact length (refer to Fig. 2c). Since the differences of all three directions are very low in the number of pillars in contact, the effect of orientation is expected to be insignificant.

\subsection{Material and Fabrication}

The textures were created in stainless steel EN-1.4301 samples with dimension of $40 \mathrm{~mm} \times 40 \mathrm{~mm}$. All the textures were produced by pulsed laser surface texturing (LST), with a picosecond laser in $400 \mathrm{kHz}$ pulse rate with maximum $50 \mathrm{~W}$ power. According to the recently published article, the LST technique is the most suitable fabrication method for deterministic surface pattern on stainless steel material [40]. In addition, the stochastic reference sample was produced by cold rolling in conventional $2 \mathrm{G}$ finish, which is used to fabricate testing samples Z001, Z002, and Z003. The surface examinations were conducted by SEM and confocal microscopy as shown in Fig. 3. The parameters of the samples are shown in the schematic diagrams (refer to Fig. 4), and details are given in Table 1.

\section{Results and Discussion}

\subsection{The Orientation Effect of the Surface Geometry}

The values of COF for each sample were calculated from five friction cycles with one sliding movement in the direction toward the subject's body. The average COF and its corresponding standard deviation were calculated from the data obtained from five strokes and tabulated in Table 2. Figure 5 shows an overview of COF measured against dry skin with all samples in three directions, $45^{\circ}$, parallel and perpendicular, with respect to the surface structure. The mean value of dynamic COF is calculated under different normal loads of $0.2 \mathrm{~N}, 0.4 \mathrm{~N}$, and $1.0 \mathrm{~N}$. Figure 5 shows the variations of $\mathrm{COF}$ associated with the sliding directions on all samples. Based on the analyzed data, the COFs of the samples Z000 (reference), Z001 (grooved channel), and Z002 (Hilbert curve) were influenced with the sliding directions, especially the samples Z000 and Z001. However, the COF of the sample Z003 (pillar) revealed insignificant variations with the sliding directions compared to the other samples. In other words, the sample Z003 (pillar) was proved to be orientation independent, and the other samples were orientation dependent. When the fingertip was sliding on the sample Z003 (pillar), the tactile friction was mainly influenced by the number of pillars in contact with skin instead of the sliding direction. The pattern layout of pillar is symmetric and identical in all directions with enough stroke length. In this case, the number of pillars in contact had a great influence of the real contact area and was determined by the texture parameters (asperity radius and spacing) and the contact radius of fingertip. According to the texture design of pillar pattern (refer to Sect. 2.1), the estimated number of pillars in contact of fingertip with all three sliding directions is very close.

In the case of perpendicular sliding against grooved channel, the friction between the skin and texture increased compared to the parallel and $45^{\circ}$ sliding direction in all three different normal loads (refer to Fig. 5). This particular phenomenon is caused by the contribution of deformation component of friction in form of hysteresis $[33,34]$. Based on the previous study of sliding direction against a grooved channel on tool steel [39], the normal force $\left(F_{\mathrm{N}}\right)$ applied on the leading edge can be determined as follows:

$F_{\mathrm{N}}=2 \int_{0}^{a} p \cdot L \cdot \mathrm{d} t$

where $p$ is the pressure along the contact area of the ridge and skin; $L$ is the length of the ridge in contact; $\mathrm{d} t=$ $\mathrm{d} l \sin \theta$ is the distance from the central axis $(t)$ between the limits of 0 and $\mathrm{a}$; and the hysteresis force $\left(F_{\text {hys }}\right)$ is the horizontal component of the force due to the applied pressure $(W)$. Based on this approach, the resulting hysteresis force $\left(F_{\text {hys }}\right)$ can be determined as follow:

$F_{\text {hys }}=W \cdot \cos \theta=\frac{F_{\mathrm{N}}}{2} \cot \theta$ 


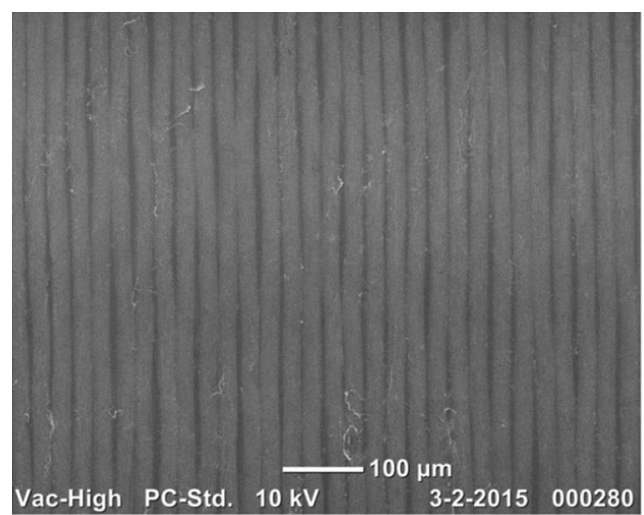

(a)

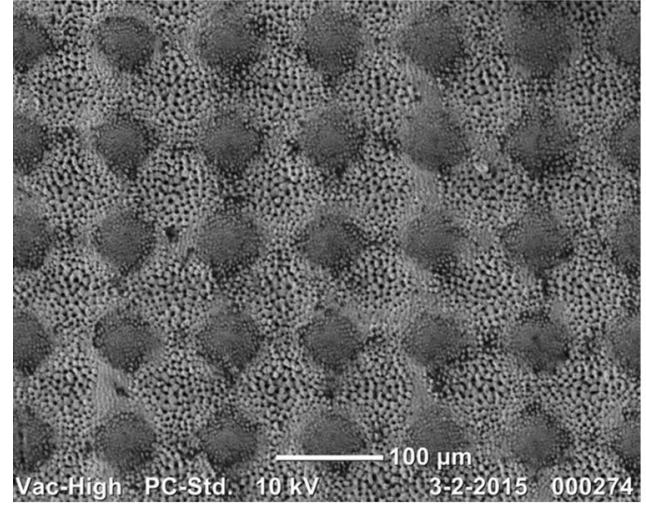

(c)

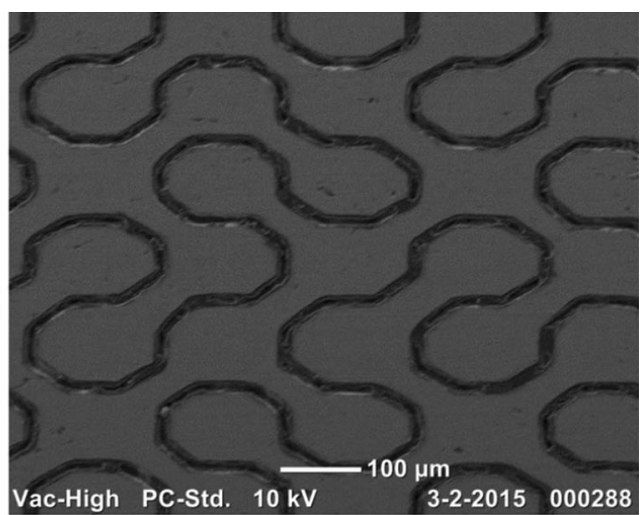

(b)

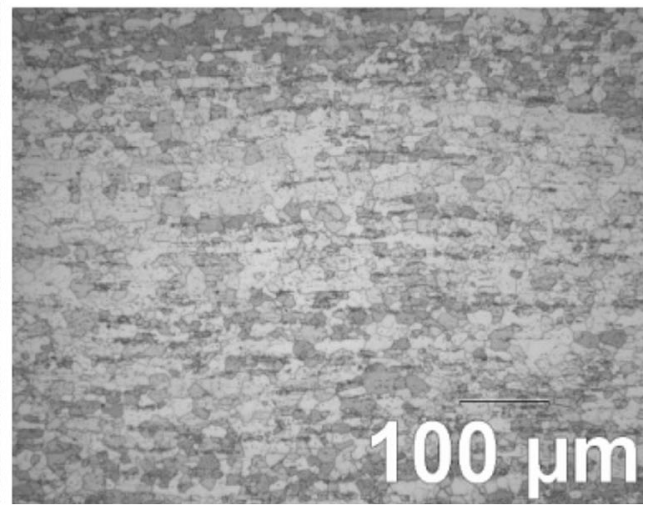

(d)

Fig. 3 Textures a grooved channel (Z001); b Hilbert curve (Z002); c pillar (Z003) examined by SEM and d reference surface with rolling direction from left to right (Z000) examined by confocal microscopy

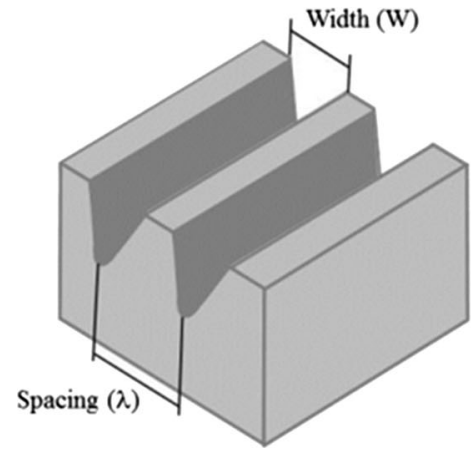

(a)

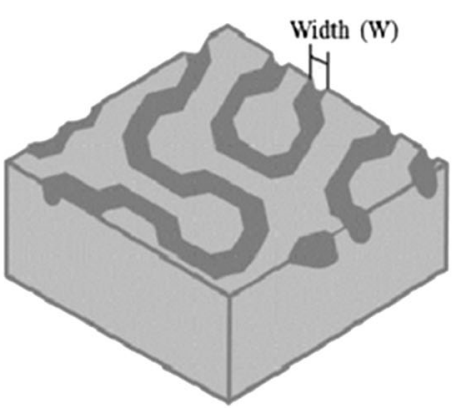

(b)

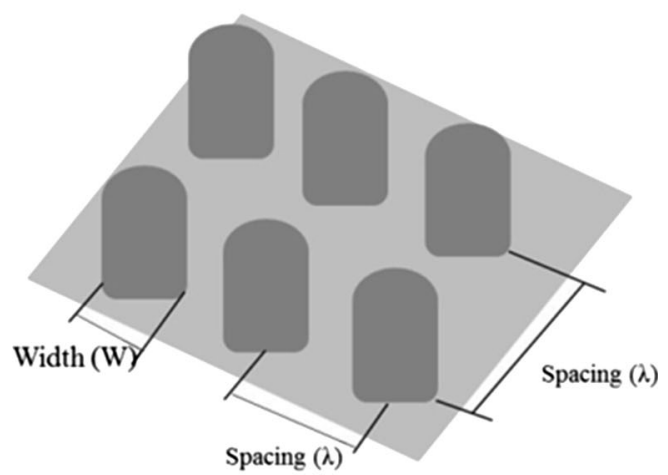

(c)

Fig. 4 Schematic drawings and parameters of a grooved channel, b Hilbert curve, and $\mathbf{c}$ pillar texture

$W$ is the force due to the applied pressure; $\theta$ is the angle between the two surfaces in contact (refer to Fig. 7).

According to the studies of skin friction at the volar forearm in contact with spherical probes [1, 19], the contribution of hysteresis to the COF ranges from 0.04 to 0.06 in the order of magnitude. Other study shows that contributions to the friction coefficient between finger and glass due to viscoelastic skin deformations can be up to 0.2 $[8,9]$. Therefore, in the case of perpendicular sliding against grooved channel texture, the role of viscoelastic skin deformation cannot be neglected and will increase overall friction between the fingertip and the counter-surface. 
Table 1 Selected surface parameters of the samples: 3D surface roughness (Sa), depth or height $(D)$, spacing $(\lambda)$, width $(W)$ and pillar radius ( $r$ ) were measured by laser scanning microscope (Keyence, VK-9700, USA)

\begin{tabular}{lllllll}
\hline Sample name & Surface texture & Sa $(\mu \mathrm{m})$ & Depth $D(\mu \mathrm{m})$ & Width $W(\mu \mathrm{m})$ & Spacing $\lambda(\mu \mathrm{m})$ & Radius $r(\mu \mathrm{m})$ \\
\hline Z001 & Grooved channel & 1.21 & 10 & 20 & 25 & - \\
Z002 & Hilbert curve & 3.43 & 15 & 20 & - & - \\
Z003 & Pillar & 4.69 & 15 & - & - & - \\
Z000 & Stochastic & 0.39 & - & - & - \\
\hline
\end{tabular}

\subsection{The Geometric Effect of the Contact area}

As shown in Fig. 6, all the textured samples (Z001, Z002, and Z003) generated less tactile friction compared to the non-textured sample (Z000), except sample Z001 in perpendicular sliding with the normal load of $1.0 \mathrm{~N}$ (the cause has been discussed in Sect. 3.1). This phenomenon was caused by the contact area between skin and counter-surface. According to the research, the reduction of the contact area can directly decrease the tactile friction [24]. In addition, the real contact area can be influenced by the contact condition which is determined by the contact ratio (Fig. 7). Westergaard developed a model to estimate the contact ratio between an elastic half-space and rigid wavy surface with spacing $(\lambda)$ and amplitude (depth or height of the structures) [36]. Study found that the Westergaard model also can be applied for a viscoelastic half-space including skin sliding on a rigid wavy surface $[11,12,16,22,26,29]$. A modified contact model was proposed in the previous study to predict the contact ratio by calculating the average contact pressure $(\bar{p})$ and the pressure needed for the fingertip under the full contact condition $\left(p^{*}\right)$ [39].

$\bar{p}=\frac{F_{N}}{\pi a^{2}}$

$p^{*}=\pi E^{*} \frac{D}{2 \lambda}$

$\frac{1}{E^{*}}=\frac{1-v_{\text {finger }}^{2}}{E_{\text {finger }}}+\frac{1-v_{\text {surface }}^{2}}{E_{\text {surface }}}$

$a=\sqrt[3]{\frac{3 R F_{\mathrm{N}}}{4 E^{*}}}$

where $F_{\mathrm{N}}$ is the applied load; $a$ is the contact radius predicted by the Hertzian equation; $E^{*}$ is the effective Young's modulus; $D$ is the depth or height of the structures (amplitude); $v_{\text {finger }}$ and $v_{\text {surface }}$ are the Poisson ratio of finger and counter-surface accordingly; $R$ is the radius of curvature of the body.

When the average contact pressure $(\bar{p})$ is smaller than the pressure required for finger undergoing the full contact condition $\left(p^{*} ; \frac{\bar{p}}{p^{*}}<1\right)$, the contact condition is considered as in partial contact. Under the partial contact condition, the skin is not deformed enough to contact the sides and valley of the texture and less real contact area is conducted between the skin and counter-surface. On the contrary case $\left(\frac{\bar{p}}{p^{*}} \geq 1\right)$, the contact condition is considered as the full contact, and the real contact area is increased due to the combination area of the surface, sides and valley of the texture.

The effective Young's modulus $\left(E^{*}\right)$ is estimated by Eq. (8) with the reported values of the Young's modulus and the Poisson ratios of skin and stainless steel $([6,14,28,33]$; refer to Table 3$)$. The radius of fingertip in contact was measured to be $8 \mathrm{~mm}$. With the estimated value of the effective Young's modulus $\left(E^{*}\right)$, the contact radius between the skin and counter-surface was estimated ( $a=3.1 \mathrm{~mm}$ ) by Eq. (9). Combined with the surface parameters of textures tabulated in Table 1 , the average contact pressure $(\bar{p})$ and the pressure needed for the fingertip under the full contact condition $\left(p^{*}\right)$ can be calculated by Eqs. (6) and (7) accordingly. Finally, the contact ratios $\left(\frac{\bar{p}}{p^{*}}\right)$ of all textured samples were predicted and results were tabulated in Table $3(\mathrm{Z} 001=0.24 ; \mathrm{Z} 002=0.48$; $\mathrm{Z} 003=0.51)$. Since the pressure ratios were less than 1 (refer to Table 3), therefore, the contact conditions can be considered as in partial contact. Under the partial contact condition, the maximum contact areas of grooved channel, Hilbert curve, and pillar textures can be derived as:

$$
\begin{aligned}
A_{\text {Groove }} & =\pi a^{2}-N w_{\text {groove }} \bar{l}_{\text {groove }} \\
& =N\left(\lambda-w_{\text {groove }}\right) \bar{l}_{\text {groove }}, \text { for } \frac{\bar{p}}{p^{*}}<1 \\
A_{\text {Hilbert }} & =\pi a^{2}-w_{\text {Hilbert }} l_{\text {Hilbert }}, \text { for } \frac{\bar{p}}{p^{*}}<1 \\
A_{\text {Grid }}= & N \pi r_{\text {Grid }}^{2}, \text { for } \frac{\bar{p}}{p^{*}}<1
\end{aligned}
$$

where $N$ is the number of grooves or pillars in contact; $w_{\text {groove }}$ is the width of the grooved channel; $\bar{l}_{\text {groove }}$ is the average length of the grooves in contact; $w_{\text {Hilbert }}$ is the width of the Hilbert curve; $l_{\text {Hilbert }}$ is the length of Hilbert curve in contact; $r_{\text {Pillar }}$ is the radius of pillar pattern. 
Table 2 Experimental data of samples in $45^{\circ}$, perpendicular and parallel sliding direction and analytical results by Westergaard method

\begin{tabular}{|c|c|c|c|c|c|c|c|}
\hline $\begin{array}{l}\text { Sliding } \\
\text { motion }\end{array}$ & Sample & $\begin{array}{l}\text { Normal load } \\
\text { (N) Mean } \pm \text { STD }\end{array}$ & $\begin{array}{l}\text { COF } \\
\text { Mean } \pm \text { STD }\end{array}$ & $\begin{array}{l}\text { Sliding } \\
\text { motion }\end{array}$ & Sample & $\begin{array}{l}\text { Normal load } \\
\text { (N) Mean } \pm \text { STD }\end{array}$ & $\begin{array}{l}\text { COF } \\
\text { Mean } \pm \text { STD }\end{array}$ \\
\hline \multirow[t]{20}{*}{$45^{\circ}$} & $\mathrm{Z} 000$ & $0.20 \pm 0.02$ & $0.61 \pm 0.05$ & \multirow{20}{*}{ Parallel } & $\mathrm{Z} 000$ & $0.22 \pm 0.04$ & $0.67 \pm 0.07$ \\
\hline & Z001 & $0.19 \pm 0.02$ & $0.50 \pm 0.01$ & & Z001 & $0.19 \pm 0.02$ & $0.41 \pm 0.09$ \\
\hline & Z002 & $0.22 \pm 0.04$ & $0.41 \pm 0.03$ & & Z002 & $0.22 \pm 0.04$ & $0.42 \pm 0.02$ \\
\hline & Z003 & $0.17 \pm 0.04$ & $0.27 \pm 0.01$ & & Z003 & $0.19 \pm 0.04$ & $0.25 \pm 0.01$ \\
\hline & $\mathrm{Z} 000$ & $0.42 \pm 0.04$ & $0.59 \pm 0.07$ & & $\mathrm{Z} 000$ & $0.43 \pm 0.07$ & $0.69 \pm 0.06$ \\
\hline & Z001 & $0.39 \pm 0.07$ & $0.44 \pm 0.05$ & & Z001 & $0.40 \pm 0.02$ & $0.47 \pm 0.08$ \\
\hline & Z002 & $0.43 \pm 0.04$ & $0.41 \pm 0.02$ & & $\mathrm{Z} 002$ & $0.39 \pm 0.07$ & $0.45 \pm 0.03$ \\
\hline & Z003 & $0.40 \pm 0.05$ & $0.24 \pm 0.01$ & & Z003 & $0.41 \pm 0.07$ & $0.24 \pm 0.01$ \\
\hline & $\mathrm{Z} 000$ & $0.61 \pm 0.03$ & $0.59 \pm 0.06$ & & $\mathrm{Z} 000$ & $0.60 \pm 0.05$ & $0.65 \pm 0.04$ \\
\hline & Z001 & $0.58 \pm 0.05$ & $0.43 \pm 0.04$ & & Z001 & $0.59 \pm 0.03$ & $0.44 \pm 0.07$ \\
\hline & Z002 & $0.61 \pm 0.03$ & $0.40 \pm 0.02$ & & Z002 & $0.58 \pm 0.07$ & $0.43 \pm 0.05$ \\
\hline & Z003 & $0.58 \pm 0.05$ & $0.25 \pm 0.01$ & & Z003 & $0.57 \pm 0.07$ & $0.23 \pm 0.02$ \\
\hline & $\mathrm{Z} 000$ & $0.79 \pm 0.05$ & $0.58 \pm 0.06$ & & $\mathrm{Z} 000$ & $0.80 \pm 0.06$ & $0.61 \pm 0.07$ \\
\hline & Z001 & $0.81 \pm 0.06$ & $0.42 \pm 0.02$ & & Z001 & $0.80 \pm 0.01$ & $0.42 \pm 0.07$ \\
\hline & Z002 & $0.78 \pm 0.03$ & $0.40 \pm 0.03$ & & Z002 & $0.79 \pm 0.05$ & $0.42 \pm 0.03$ \\
\hline & Z003 & $0.81 \pm 0.05$ & $0.23 \pm 0.02$ & & Z003 & $0.81 \pm 0.05$ & $0.24 \pm 0.03$ \\
\hline & $\mathrm{Z} 000$ & $1.03 \pm 0.05$ & $0.57 \pm 0.05$ & & $\mathrm{Z} 000$ & $0.92 \pm 0.12$ & $0.56 \pm 0.04$ \\
\hline & Z001 & $1.00 \pm 0.07$ & $0.41 \pm 0.03$ & & Z001 & $1.02 \pm 0.04$ & $0.42 \pm 0.04$ \\
\hline & Z002 & $0.88 \pm 0.09$ & $0.38 \pm 0.03$ & & Z002 & $0.90 \pm 0.09$ & $0.42 \pm 0.02$ \\
\hline & Z003 & $1.00 \pm 0.15$ & $0.24 \pm 0.02$ & & Z003 & $0.96 \pm 0.12$ & $0.24 \pm 0.01$ \\
\hline \multirow[t]{20}{*}{ Perpendicular } & $\mathrm{Z} 000$ & $0.20 \pm 0.02$ & $0.67 \pm 0.07$ & \multirow{20}{*}{ Westergaard } & $\mathrm{Z} 000$ & - & - \\
\hline & Z001 & $0.19 \pm 0.01$ & $0.51 \pm 0.05$ & & Z001 & 0.20 & 0.45 \\
\hline & Z002 & $0.18 \pm 0.03$ & $0.43 \pm 0.02$ & & Z002 & 0.20 & 0.54 \\
\hline & Z003 & $0.18 \pm 0.04$ & $0.26 \pm 0.01$ & & Z003 & 0.20 & 0.27 \\
\hline & $\mathrm{Z} 000$ & $0.40 \pm 0.03$ & $0.60 \pm 0.05$ & & $\mathrm{Z} 000$ & - & - \\
\hline & Z001 & $0.39 \pm 0.01$ & $0.50 \pm 0.07$ & & Z001 & 0.40 & 0.36 \\
\hline & Z002 & $0.39 \pm 0.07$ & $0.42 \pm 0.02$ & & $\mathrm{Z} 002$ & 0.40 & 0.41 \\
\hline & Z003 & $0.43 \pm 0.05$ & $0.26 \pm 0.01$ & & Z003 & 0.40 & 0.26 \\
\hline & $\mathrm{Z} 000$ & $0.61 \pm 0.03$ & $0.55 \pm 0.05$ & & $\mathrm{Z} 000$ & - & - \\
\hline & Z001 & $0.61 \pm 0.02$ & $0.50 \pm 0.05$ & & Z001 & 0.60 & 0.32 \\
\hline & Z002 & $0.59 \pm 0.05$ & $0.43 \pm 0.04$ & & Z002 & 0.60 & 0.36 \\
\hline & Z003 & $0.60 \pm 0.05$ & $0.26 \pm 0.02$ & & Z003 & 0.60 & 0.25 \\
\hline & $\mathrm{Z} 000$ & $0.79 \pm 0.05$ & $0.58 \pm 0.06$ & & $\mathrm{Z} 000$ & - & - \\
\hline & Z001 & $0.78 \pm 0.03$ & $0.51 \pm 0.06$ & & Z001 & 0.80 & 0.30 \\
\hline & Z002 & $0.79 \pm 0.06$ & $0.42 \pm 0.04$ & & Z002 & 0.80 & 0.33 \\
\hline & Z003 & $0.78 \pm 0.04$ & $0.27 \pm 0.01$ & & Z003 & 0.80 & 0.25 \\
\hline & $\mathrm{Z} 000$ & $1.02 \pm 0.04$ & $0.48 \pm 0.04$ & & $\mathrm{Z} 000$ & - & - \\
\hline & Z001 & $1.01 \pm 0.04$ & $0.53 \pm 0.02$ & & Z001 & 1.00 & 0.29 \\
\hline & Z002 & $0.93 \pm 0.11$ & $0.41 \pm 0.02$ & & Z002 & 1.00 & 0.31 \\
\hline & Z003 & $0.99 \pm 0.13$ & $0.26 \pm 0.01$ & & $\mathrm{Z} 003$ & 1.00 & 0.24 \\
\hline
\end{tabular}

Now, a modified Westergaard model can be used to describe the contact width $2 a_{\text {westergaard }}$.

$$
2 a_{\text {westergaard }}=\frac{2 \lambda}{\pi} \sin ^{-1}\left(\frac{p}{p^{*}}\right)^{1 / 2}
$$

where $\lambda$ is spacing (or wavelength); $p$ is mean surface pressure; $p^{*}$ is the pressure needed for full continuous contact.

With the value of contact ratio $\left(\frac{p}{p^{*}}\right.$; refer to Table 3$)$ and known spacing $\lambda$ of each texture (refer to Table 1 ), the 


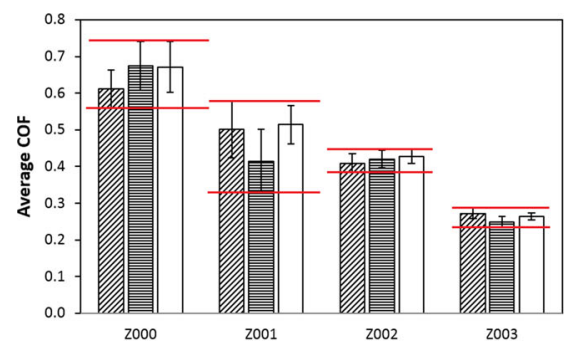

(a)

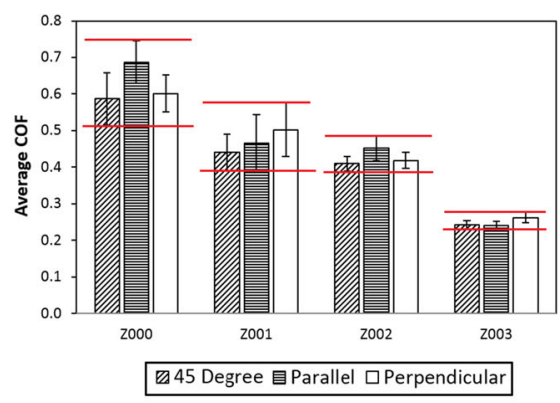

(b)

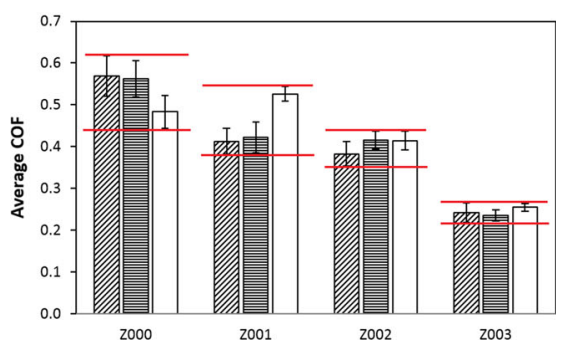

(c)

Fig. 5 Overview of COF versus sliding direction for each sample with different normal load a $0.2 \mathrm{~N}, \mathbf{b} 0.4 \mathrm{~N}$, and $\mathbf{c} 1.0 \mathrm{~N}$; Redline indicates the variation of $\mathrm{COF}$ with sliding directions (Color figure online)

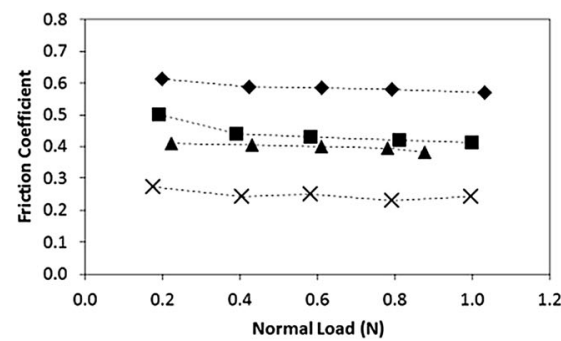

(a)

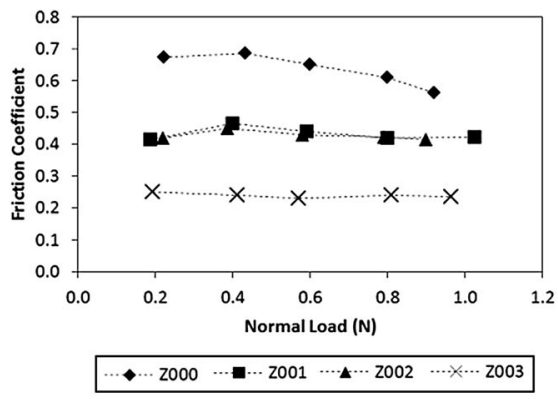

(b)

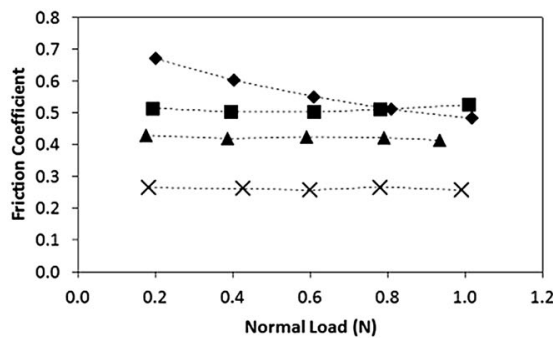

(c)
Fig. 6 Values of COF versus normal load for each sample in a $45^{\circ}$, b parallel, and c perpendicular sliding. The ranges of COF were $0.27-0.61,0.19-1.02$, and $0.18-1.02$ for all samples (including reference sample) in the $45^{\circ}$, parallel, and perpendicular sliding directions accordingly. The standard deviations of all data plots ranged from 0.01 to 0.09 . The detailed data are shown in Table 3
Fig. 7 Schematic diagram of ridge-texture contact, the upper body is the single fingerprint ridge against the textured counter-body

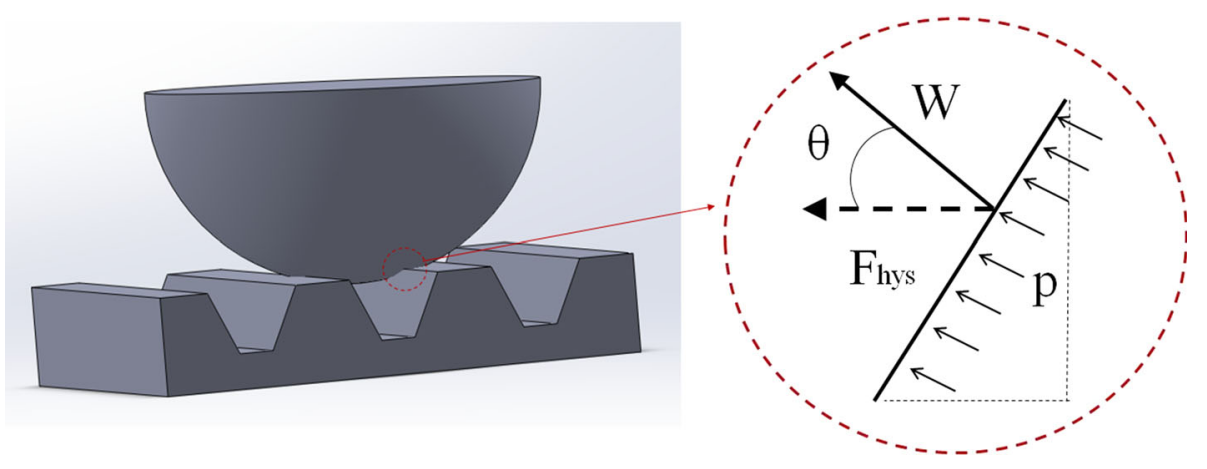

contact width $2 a_{\text {westergaard }}$ can be calculated. As the result, the contact area of each textured samples need to be calculated (refer to Eqs. 10, 11, 12). According to Eq. (3), the functional forms of COF between the skin and textured counter-surface can be calculated by inputting the values of the intrinsic interfacial shear strength $\tau_{0}$ and the pressure coefficient $\alpha$ which are reported in the literature as $5.5 \mathrm{kPa}$ and 0.23 accordingly (refer to Table 3; [10, 33]). The normal loads $\left(F_{\mathrm{N}}\right)$ were $0.2 \mathrm{~N}, 0.4 \mathrm{~N}, 0.6 \mathrm{~N}, 0.8 \mathrm{~N}$, and $1.0 \mathrm{~N}$, and the results of estimated COF were charted. As shown in Fig. 8, the values of estimated COF correspond well with the range of $\mathrm{COF}$ obtained from friction measurements for the all samples, especially for the sample
Z003 (pillar). For example, under the normal load of $0.2 \mathrm{~N}$, the estimated COF of the sample Z003 was 0.28 compared with the calculated $\mathrm{COF}$ from the friction measurements $\left(0.27\right.$ for $45^{\circ}, 0.25$ for parallel, and 0.26 for the perpendicular sliding direction; refer to Fig. 8c).

Overall, the sample Z003 (pillar) showed a better performance compared to the other samples in friction reduction from both experimental and analytical results (refer to Figs. 6, 8). Moreover, based on the experimental results, the sample Z003 (pillar) was orientation independent, while the other samples were orientation dependent in $45^{\circ}$, parallel, and perpendicular sliding directions. With the respect to the texture designs based on the friction model 


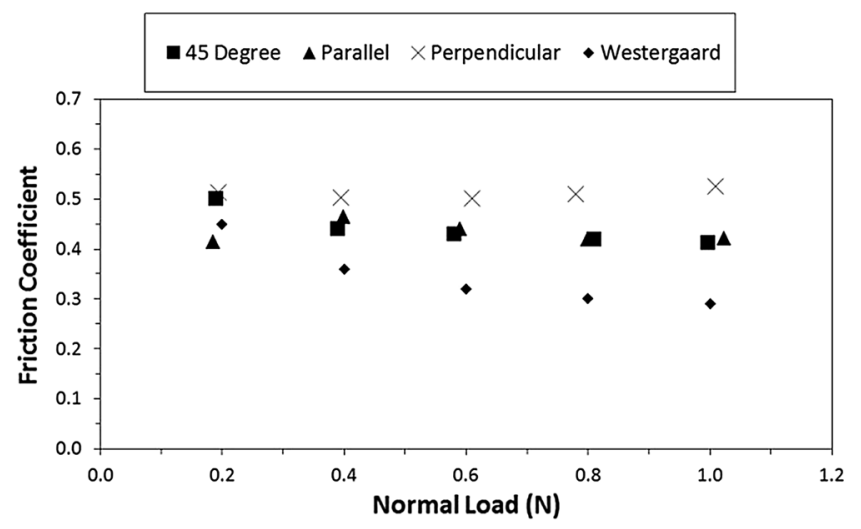

(a)

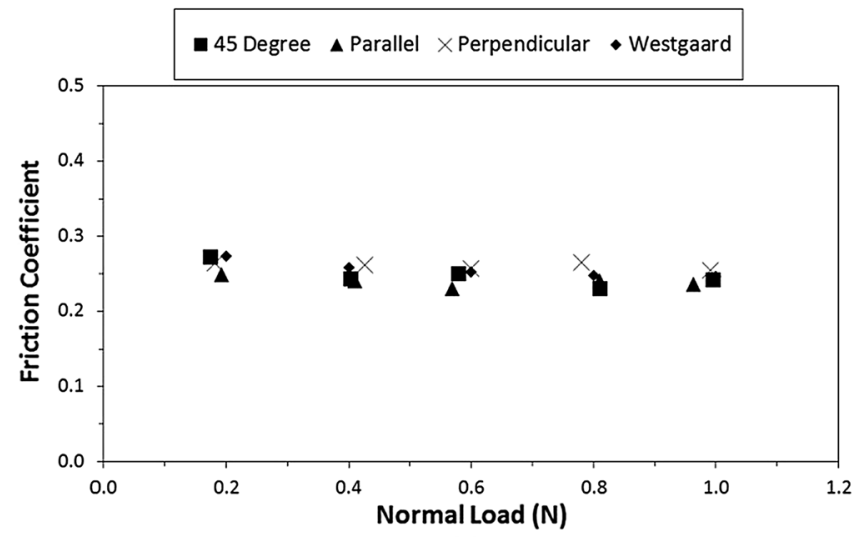

(c)

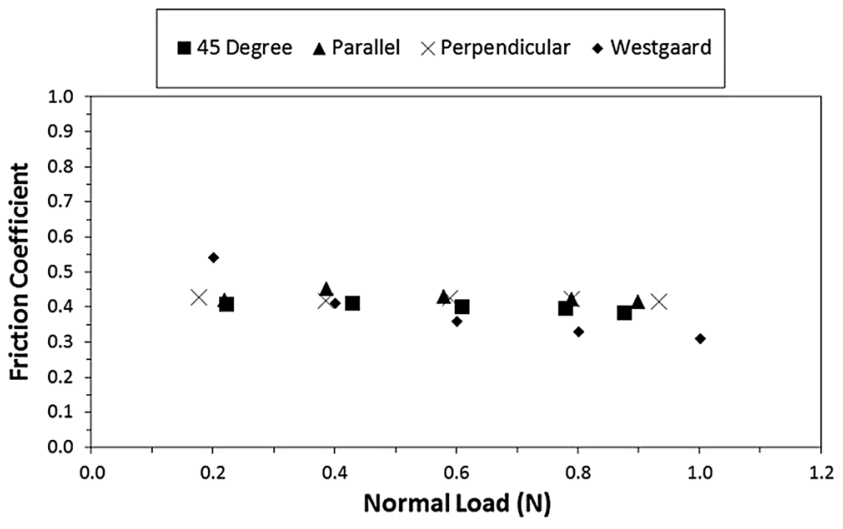

(b)

Fig. 8 Values of COF (both experimental and analytical results) versus normal load for a sample Z001 (grooved channel), b sample Z002 (Hilbert curve), and c sample Z003 (pillar). The detailed values are listed in Table 2

Table 3 Parameters from literature were used to calculate the pressure ratios and COF of textured samples [6, 14, 28, 33]), and the normal load $\mathrm{F}_{\mathrm{N}}$ (maximum value) was obtained from the skin friction measurements

\begin{tabular}{llll}
\hline Material property & Values & Sample & Pressure ratio \\
\hline$E_{\text {finger }}$ & $0.2 \mathrm{MPa}$ & Z001 & 0.24 \\
$E_{\text {surface }}$ & $150 \mathrm{GPa}$ & Z002 & 0.48 \\
$v_{\text {finger }}$ & 0.48 & Z003 & 0.51 \\
$v_{\text {surface }}$ & 0.3 & & \\
$F_{\mathrm{N}}$ & $1.0 \mathrm{~N}$ & & \\
Finger radius, $R$ & $8 \mathrm{~mm}$ & & \\
Pressure coefficient $\alpha$ & 0.23 & & \\
Intrinsic interfacial shear strength $\tau_{0}$ & $5.5 \mathrm{kPa}$ &
\end{tabular}

and orientation effect, the pillar pattern showed superior performance compared to the grooved channel and Hilbert curve pattern and reference sample.

\section{Conclusions}

Based on the experimental results, less friction forces were observed on the textured stainless steel samples (Z001, Z002, and Z003) compared to non-textured sample in three sliding directions (perpendicular, parallel, and $45^{\circ}$ ) except sample Z001 in perpendicular sliding with the normal load of $1.0 \mathrm{~N}$. In the concern of friction reduction, the pillar (Z003) texture has the advantage over Hilbert curve and grooved channel textures in decreasing the friction force under the partial contact condition $\left(\frac{\bar{p}}{p^{*}}<1\right)$. From the analytical approach, the pillar (Z003) also showed lower values of COF compared to the other samples, which was consistent with the experimental results. 
Furthermore, the influence of orientation effect was insignificant for pillar (Z003) textures due to the geometrical advantage. However, the sliding directions affected other samples, especially the perpendicular sliding for the grooved channel (Z001) and stochastic surface (Z000). In the case of sample Z001 in perpendicular sliding, the resulting hysteresis force was able to increase the deformation component of friction between the skin and the leading edges of counter-surface, and as a result, the overall friction was increased.

From both the experimental and analytical approaches, the pillar pattern was proved to be the optimal textural design in the concern of friction reduction and orientation effect. The pillar structure can greatly reduce the dynamic friction between the skin and counter-surface without the influence of sliding directions $\left(45^{\circ}\right.$, parallel, and perpendicular) compared to the other samples.

Acknowledgements This work was supported by the Research Programme of the Research Fund for Coal and Steel, Contract No. RFSR-CT-2011-00022.

Open Access This article is distributed under the terms of the Creative Commons Attribution 4.0 International License (http://crea tivecommons.org/licenses/by/4.0/), which permits unrestricted use, distribution, and reproduction in any medium, provided you give appropriate credit to the original author(s) and the source, provide a link to the Creative Commons license, and indicate if changes were made.

\section{References}

1. Adams, M.J., Briscoe, B.J., Johnson, S.A.: Friction and lubrication of human skin. Tribol. Lett. 26(3), 239-253 (2007)

2. Butz, A.R.: Space filling curves and mathematical programming. Inf. Control 12, 314-330 (1968)

3. Butz, A.R.: Convergence with Hilbert's space filling curve. J. Comput. Syst. Sci. 3, 128-146 (1969)

4. Chen, X., Bames, C.J., Childs, T.H.C., Henson, B., Shao, F.: Materials' tactile testing and characterization for consumer products' affective packaging design. Mater. Des. 30(10), 4299-4310 (2009)

5. Braun, D., Greiner, C., Schneider, J., Gumbsch, P.: Efficiency of laser surface texturing in the reduction of friction under mixed lubrication. Tribol. Int. 77, 142-147 (2014)

6. Dandekar, K., Raju, B.I., Srinivasan, M.A.: 3-D finite-element models of human and monkey fingertips to investigate the mechanics of tactile sense. J. Biomech. Eng. Trans. ASME 125(5), 682-691 (2003)

7. Derler, S., Gerhardt, L.-C.: Tribology of skin: review and analysis of experimental results for the friction coefficient of human skin. Tribol. Lett. 45, 1-27 (2012)

8. Derler, S., Gerhardt, L.-C., Lenz, A., Bertaux, E., Hadad, M.: Friction of human skin against smooth and rough glass as a function of the contact pressure. Tribol. Int. 42, 1565-1574 (2009)

9. Derler, S., Huber, R., Feuz, H.P., Hadad, M.: Influence of surface microstructure on the sliding friction of plantar skin against hard substrates. Wear 267(5-8), 1281-1288 (2009)
10. Derler, S., Suess, J., Rao, A., Rotaru, G.-M.: Influence of variations in the pressure distribution on the friction of the finger pad. Tribol. Int. 63, 14-20 (2013)

11. Duvefelt, K., Olofsson, U., Johannesson, C.M., Skedung, L.: Model for contact between finger and sinusoidal plane evaluate adhesion and deformation component of friction. Tribol. Int. 96, 389-394 (2016)

12. Greenwood, J.A., Tabor, D.: The friction of hard sliders on lubricated rubber: the importance of deformation losses. Proc. Phys. Soc. 71, 989-1001 (1958)

13. Griffiths, J.G.: Table-driven algorithms for generating spacefilling curves. Comput. Aided Des. 17, 37-41 (1985)

14. Greaves, G.N., Greer, A.L., Lakes, R.S., Rouxel, T.: Poisson's ratio and modern materials. Nat. Mater. 10, 823-837 (2011)

15. Hilbert, D.: Ueber die stetige Abbildung einer Linie auf ein Flaechenstueck. Math. Ann. 38, 459-460 (1891)

16. Johnson, K.L., Greenwood, J.A., Higginson, J.G.: The contact of elastic regular wavy surface. Int. J. Mech. Sci. 6, 383-396 (1985)

17. Kang, M., Park, Y.M., Kim, B.H., Seo, Y.H.: Micro- and nanoscale surface texturing effects on surface friction. Appl. Surf. Sci. 345, 344-348 (2015)

18. Kim, M.S., Kim, I.Y., Park, Y.K., Lee, Y.Z.: The friction measurement between finger skin and material surfaces. Wear 301, 338-342 (2013)

19. Kwiatkowska, M., Franklin, S.E., Hendriks, C.P., Kwiatkowski, K.: Friction and deformation behaviour of human skin. Wear 267(5-8), 1264-1273 (2009)

20. Lewis, R., Carre, M.J., Tomlinson, S.E.: Skin friction at the interface between hands and sports equipment. Proc. Eng. 72, 611-617 (2014)

21. Maeno, T., Kobayashi, K., Yamazaki, N.: Relationship between the structure of human finger tissue and the location of tactile receptors. JSME Int. J. Ser. C Mech. Syst. Mach. Elem. Manuf. 41(1), 94-100 (1998)

22. Menga, N., Putignano, C., Carbone, G., Demelio, G.P.: The sliding contact of a rigid wavy surface with a viscoelastic halfspace. Proc. R. Soc. A Math. Phys. Eng. Sci. 470, 1-14 (2014)

23. Pasumarty, S.M., Johnson, S.A., Watson, S.A., Adams, M.J.: Friction of the human finger pad: influence of moisture, occlusion and velocity. Tribol. Lett. 44, 117-137 (2011)

24. Prodanov, N., Gachot, C., Rosenkanz, A., Muchlich, F., Muser, M.H.: Contact mechanics of laser-textured surfaces. Tribol. Lett. 50, 41-48 (2013)

25. Peano, G.: Sur une coube, qui remplit toute une aire plaine. Math. Ann. 36, 157-160 (1890)

26. Prokopovich, P., Perni, S.: Adhesion models: from single to multiple asperity contacts. Colloids Surf. A 383, 95-101 (2011)

27. Schreiner, S., Rechberger, M., Bertling, J.: Haptic perception of friction-correlating friction measurements of skin against polymer surfaces with subjective evaluations of the surfaces' grip. Tribol. Int. 63, 21-28 (2013)

28. Shao, F., Childs, T.H.C., Barnes, C.J., Henson, B.: Finite element simulations of static and sliding contact between a human fingertip and textured surfaces. Tribol. Int. 43, 2308-2316 (2010)

29. Skedung, L., Danerlov, K., Olofsson, U., Johannesson, C.M., Aikala, M., Kettle, J., Arvidsson, M., Berglund, B., Rutland, M.W.: Tactile perception: Finger friction, surface roughness and perceived coarseness. Tribol. Int. 44, 505-512 (2011)

30. Soneda, T., Nakano, K.: Investigation of vibrotactile sensation of human finger-pads by observation of contact zones. Tribol. Int. 43, 210-217 (2010)

31. Suh, M., Chae, Y., Kim, S., Hinoki, T., Kohyama, A.: Effect of geometrical parameters in micro-grooved crosshatch pattern under lubricated sliding friction. Tribol. Int. 43, 1508-1517 (2010) 
32. Tagami, H., Yoshikuni, K.: Interrelationship between water-barrier and reservoir functions of pathologic stratum corneum. Arch. Dermatol. 121, 642-645 (1985)

33. Tomlinson, S.E., Carre, M.J., Lewis, R., Franklin, S.E.: Human finger contact with small, triangular ridged surfaces. Wear 271, 2346-2353 (2011)

34. Tomlinson, S.E., Lewis, R., Carre, M.J.: The effect of normal force and roughness on friction in human finger contact. Wear 267(5-8), 1311-1318 (2009)

35. Van Kuilenburg, J., Masen, M.A., Groenendijk, M.N.W., Bana, V., van der Heide, E.: An experimental study on the relation between surface texture and tactile friction. Tribol. Int. 48, 15-21 (2012)

36. Westergaard, H.M., Bearing pressures and cracks. J .Appl. Mech. Trans. ASME 6, 49-53 (1939)

37. Wolfram, L.J.: Friction of skin. J. Soc. Cosmet. Chem. 34(8), 465-476 (1983)
38. Yuan, Y., Verma, R.: Measuring microelastic properties of stratum corneum. Colloids Surf. B 48, 6-12 (2006)

39. Zhang, S., Rodriguez Urribarri, A., Morales Hurtado, M., Zeng, X., van der Heide, E.: The role of the sliding direction against a grooved channel texture on tool steel: an experimental study on tactile friction. Int. J. Solids Struct. 56-57, 53-61 (2015)

40. Zhang, S., Zeng, X., Matthews, D.T.A., Igartua, A., RodriguezVidal, E., Contreras Fortes, J., Saenz de Viteri, V., Pagano, F., Wadman, B., van der Heide, E.: Selection of micro-fabrication techniques on stainless steel sheet for skin friction. Friction 4(2), 89-104 (2016)

41. Zhang, S., Zeng, X., Matthews D.T.A., Igartua, A., RodriguezVidal, E., Contreras Fortes, J., van der Heide, E.: Texture design for light touch perception. Biosurf. Biotribol. 3(1), 25-34 (2017) 